\title{
Association épisodique d'halophytes stricts et de glycophytes dans un écosystème hydromorphe salé en zone semi-aride
}

\author{
C Abdelly ${ }^{1}$, M Lachaal ${ }^{1}$, C Grignon 2*, A Soltani ${ }^{1}$, M Hajji ${ }^{3}$ \\ ' INRST, unité de biotechnologie végétale et de ressources génétiques, nutrition minérale, BP 95, 2050 Hammam Lif, Tunisie; \\ 2 ENSA-INRA, biochimie et physiologie végétales (CNRS-URA 573), F34060 Montpellier cedex 1, France; \\ ${ }^{3}$ Faculté des sciences de Tunis, physiologie végétale, campus universitaire, 1060 Tunis, Tunisie
}

(Reçu le 15 janvier 1995 ; accepté le 17 août 1995)

\begin{abstract}
Résumé - La production de biomasse et le prélèvement d'éléments nutritifs majeurs $(N, P, K)$ dans une parcelle mise hors pâturage en bordure de la sebkha d'Enfidha (100 km au sud de Tunis, étage bioclimatique semi-aride inférieur) ont été suivis au cours de 2 années consécutives, l'une particulièrement sèche et l'autre relativement pluvieuse. Environ la moitié de la biomasse aérienne sur la parcelle correspond à des halophytes pérennes caractéristiques de sols à forte salinité. Au cours de l'année pluvieuse, $40 \%$ de la production primaire de l'écosystème sont dus aux espèces annuelles, parmi lesquelles Medicago ciliaris (L) Krock, $M$ polymorpha L, $M$ truncatula Gaertn, et $M$ minima Grufb sont dominantes. La réaction de ces Medicago au stress salin a été étudiée au laboratoire. Au stade végétatif, la production de MS est restreinte par $\mathrm{NaCl}(110 \mathrm{mM}$ et $160 \mathrm{mM})$ chez tous les Medicago. Cet effet est lié à une réduction de la surface foliaire plutôt qu'à une baisse de la vitesse d'assimilation nette. Le sel diminue la production de graines en limitant le nombre de gousses, sans affecter le nombre de graines par gousse ni le poids individuel de la graine. La viabilité des graines n'est que très faiblement affectée. En dépit de leur origine (bordure de sebkha peuplée d'halophytes), les Medicago se sont révélés relativement sensibles à la salinité. La dynamique de leur reproduction dépend du maintien de la salinité à un niveau faible. Ces résultats suggèrent que ces plantes exploitent un horizon superficiel moins salé que celui qui supporte la croissance des halophytes. La présence des halophytes pourrait favoriser le maintien d'un horizon peu salé et relativement fertile, occupé périodiquement par les Medicago annuels.
\end{abstract}

\section{Medicago / association halophytes-glycophytes / production de biomasse / salinité / aridité}

Summary - Episodic association of strict halophytes and glycophytes in a saline, hydromorphic ecosystem in semi-arid zones. The biomass production and the uptake of mineral nutrients $(N, P, K)$ in an ungrazed area edging the sebkha of Enfidha (100 km south-east of Tunis; semi-arid bioclimatic zone) were studied for 2 successive years; the first year was particularly dry, the second relatively rainy. Half the aerial biomass was produced by perennial halophytes. Annual plants were responsible for a large part of the ecosystem productivity in the second year. Among them, Medicago ciliaris $(L)$ Krock, M polymorpha $L$, M truncatula Gaertn and $\mathrm{M}$ minima Grufb were the dominant species (40\% of ecosystem primary production). We studied their response to salinity in controlled culture conditions. Vegetative growth (dry matter production) was reduced by $\mathrm{NaCl}(110$ or $160 \mathrm{mM}$ ) in all Medicago species. This effect was related to a reduction in leaf area rather than in assimilation efficiency. The number of seeds produced per plant and per unit biomass was severely reduced in the presence of salt. In contrast, individual seed weight, as well as their

* Correspondance et tirés à part 
germinating power, were only weakly affected. In spite of their origin (edge of sebkha crowded by halophytes), the Medicago species studied appeared to be glycophytic, on the basis of their growth and nutritional responses to $\mathrm{NaCl}$. Their reproduction depended on the maintenance of the salt concentration at low levels. These results suggest that these plants exploit the upper horizon layer, which is less salty than the deeper ones, which support the halophytes. The presence of halophytes might contribute to maintaining a fertile and less salty superficial layer, occupied periodically by annual Medicago species.

\section{Medicago / halophyte-glycophyte association / biomass production / salinity / aridity}

\section{INTRODUCTION}

La superficie des sols salés, estimée dans le monde à plus de 900 millions d'hectares (Epstein, 1980 ; Epstein et al, 1980), est en progression continue. Ces vastes étendues ne sont généralement pas exploitées, excepté lorsqu'elles constituent occasionnellement des pâturages médiocres, ou des périmètres irrigués à faibles rendements (77 millions d'hectares, soit $1 / 3$ des surfaces irriguées) (Epstein, 1980). Cette situation est préoccupante dans la plupart des pays en voie de développement où les terres fertiles et les eaux de bonne qualité sont devenues nettement insuffisantes pour une population de plus en plus nombreuse (Shay, 1990). Près de 15 millions d'hectares sont affectés par la salinité dans le Maghreb et le Moyen-Orient (Le Houérou, 1986), dont environ $1 / 6$ sont occupés par des chotts et sebkhas (dépressions salées, épisodiquement inondées : chott désigne les systèmes de grande étendue, sebkha est réservée aux dépressions de taille plus réduite) colonisés par des halophytes. En Tunisie, ces régions correspondent aux plaines inondables mal drainées, aux dépressions fermées, aux marais côtiers, à certains cônes d'épandages, aux bourrelets éoliens en bordures de sebkhas, et enfin aux oasis (Novikoff, 1961).

Dans les sebkhas, où le sol est fortement salé (avec une prédominance des chlorures) et où l'hydromorphie est totale, les associations Suaeda, Salicornia, Arthrocnemum et Halocnemum dominent. Ces halophytes à feuilles succulentes ne représentent pas une ressource pastorale réelle, tout au moins pour les ovins, probablement parce qu'ils sont très chargés en sel. Par exemple, dans les parties aériennes de l'Halocnemum strobilaceum (Pall) $M$ Bieb, qui colonise les zones les plus salées (plus de 60 milliSiemens par $\mathrm{cm}$ ), la charge ionique est excessive, et qualitativement déséquilibrée : $\mathrm{NaCl}$ représente 15 à $30 \%$ de la matière sèche (MS), et $\mathrm{K}^{+}$seulement $1 \%$ (Smiti, 1987), ce qui correspond à une concentration ionique moyenne dans l'eau des tissus de 1,5 à 3
M. A titre de comparaison, les luzernes contiennent 10 fois moins de $\mathrm{NaCl}$, mais 4 à 5 fois plus de $\mathrm{K}^{+}$, et la concentration ionique globale y reste de l'ordre de 0,4 M (Abdelly, 1992).

Dans le cas des périphéries de sebkhas, où le niveau de salinité varie fortement suivant les horizons, les halophytes cohabitent avec diverses annuelles, qui sont bien appréciées par le bétail. Dans une première étape, nous avons étudié les potentialités de production de matière sèche de ces plantes dans leur biotope naturel, et les prélèvements qu'elles opèrent. Ceci nous a permis d'identifier les principales espèces déterminantes dans le bilan des éléments nutritifs majeurs carbone, azote, potassium et phosphore. Les plantes repérées ont été ensuite soumises, dans des conditions contrôlées, au stress salin caractéristique de leur habitat naturel afin de préciser les traits physiologiques qui leur permettent de coloniser la bordure de sebkha. Notre étude s'inscrit dans le cadre d'un programme de recherche pour améliorer la productivité des terrains salés par le contrôle du peuplement végétal, en implantant des espèces tolérant les stress de ces milieux. Nous avons accordé un intérêt particulier aux légumineuses, espèces majoritaires dans le groupe des annuelles, en raison de leur qualité fourragère et de leur capacité d'améliorer le budget azoté des sols salés.

\section{MATÉRIELS ET MÉTHODES}

\section{Étude sur le terrain}

Une parcelle expérimentale de $1000 \mathrm{~m}^{2}$ a été mise hors pâturage dans le domaine des terres relevant de l'Office des terres domaniales d'Enfidha (100 km qu sud-est de Tunis). Cette station présente une faible pente du nord-ouest au sud-est, et débouche sur une sebkha communiquant avec la mer. Elle appartient à l'étage bioclimatique semi-aride inférieur à hiver doux recevant une hauteur moyenne de pluie de $300 \mathrm{~mm}$ par an (Nasr et al, 1989). La production de biomasse a été suivie en 1988 et 1989 par 3 prélèvements par an. En 1988, les pluies ont été irrégulières et peu abon- 
dantes (235 mm), mais la hauteur de pluie de 1989 s'est élevée à $423 \mathrm{~mm}$.

\section{Expériences de laboratoire}

Les expériences ont porté sur 4 espèces de Medicago, $M$ ciliaris (L) Krock, $M$ polymorpha L, $M$ truncatula Gaertn et $M$ minima Grufb. Les graines proviennent de la station d'Enfidha. Les plantes sont cultivées, jusqu'à la maturation des fruits, dans des pots de terre cuite remplis d'un sol ayant un niveau de fertilité voisin (d'après les analyses des éléments minéraux majeurs) de celui de la station où ont été prélevées les graines. Après $28 \mathrm{j}$ de culture sur milieu témoin (eau de ville titrant $10 \mathrm{mM} \mathrm{NaCl}$ ), les plantes sont irriguées, 1 à 2 fois par semaine, avec cette eau de ville additionnée de 0,6 ou 9 g.l-1 de $\mathrm{NaCl}$ (milieux T, T6 et T9, contenant $\mathrm{NaCl} 10,110$ et $160 \mathrm{mM}$ ).

Les cultures sont conduites sous serre vitrée, la température et l'humidité relative sont en moyenne de $26^{\circ} \mathrm{C}$ et $50 \%$ le jour et $20^{\circ} \mathrm{C}$ et $70 \%$ la nuit. Deux récoltes sont faites. La première, au début de la floraison, correspond à la production maximale de matière sèche. La seconde, à la maturation des fruits, permet d'estimer le rendement en graines. Parallèlement à ces expériences sur sol, les mêmes espèces sont cultivées en milieu hydroponique sur une solution de base de Long Ashton modifiée (Hewitt, 1966). Après 3 sem, une partie des plantes est transférée sur milieux additionnés de $\mathrm{NaCl} 6$ ou $12 \mathrm{~g} . \mathrm{l}^{-1}$. La récolte a lieu 3 sem plus tard.

La biomasse et l'accumulation d'éléments minéraux dans la parcelle observée sont suivies à l'aide de la méthode suivante : pour les espèces pérennes, le dénombrement complet et les mensurations portent sur des placettes de $25 \mathrm{~m}^{2}$. Pour éviter de perturber le système, les prélèvements pour les mesures de biomasse et de composition élémentaire sont faits sur des plantes homologues situées autour de ces placettes d'observation. Le dénombrement des annuelles est accompagné du prélèvement de l'ensemble des individus de ces différentes espèces, sur des surfaces de $3 \mathrm{~m}^{2}$ ( 6 placettes de $0,5 \mathrm{~m}^{2}$ ). La production annuelle ou primaire de matière sèche des halophytes pérennes est estimée selon la méthode de Berger et al
(1978), par la différence entre les biomasses mesurées en début (hiver) et fin de saison (été-automne) ; chez les annuelles, elle correspond à la récolte de printemps.

Les prélèvements de sol ont été faits au printemps. Des sondages entre les touffes d'halophytes vers le centre de la parcelle ont permis de collecter des échantillons tous les $20 \mathrm{~cm}$ entre la surface du sol et $1 \mathrm{~m}$ de profondeur. II s'agit d'un échantillon moyen par horizon (mélange de 5 carottes prélevées sur une superficie de $1 \mathrm{~m}^{2}$ ). Sur chaque échantillon moyen, des analyses ont porté sur 8 répétitions (cf légende tableau I).

\section{Mesures}

Les masses de matière sèche (MS) sont mesurées après $48 \mathrm{~h}$ à l'étuve à $80^{\circ} \mathrm{C}$. La croissance moyenne relative (CMR) entre 2 prélèvements aux jours $t_{1}$ et $t_{2}$ est calculée par :

$$
C M R=\left(M S_{2}-M S_{1}\right) / M S^{*}\left(t_{2}-t_{1}\right)
$$

avec $M S=\left(M S_{2}-M S_{1}\right) / \ln \left(M S_{2} / M S_{1}\right)$ (moyenne logarithmique) (Hunt, 1990). Les surfaces foliaires sont mesurées par découpage et pesée des images des feuilles photocopiées. L'indice de surface foliaire ISF est la surface foliaire associée à $1 \mathrm{~g}$ MS totale de plante $\left(\mathrm{cm}^{2} \cdot \mathrm{g}^{-1} \mathrm{MS}\right)$. La vitesse d'assimilation nette par unité de surface foliaire ANSF est obtenue en ramenant la vitesse moyenne de production de matière sèche entre $t_{1}$ et $t_{2}$ à la surface foliaire moyenne sur cet intervalle:

$$
A N S F=\left(M S_{2}-M S_{1}\right) / S F^{*}\left(t_{2}-t_{1}\right)
$$

où $S F=\left(S F_{2}-S F_{1}\right) / \ln \left(S F_{2} / S F_{1}\right)$

Les dosages ont été faits sur des échantillons réduits en poudre fine. $\mathrm{Na}^{+}, \mathrm{K}^{+}, \mathrm{Ca}^{2+}$ ont été dosés en émission de flamme (spectrophotomètre Eppendorf) sur le produit d'une extraction à $\mathrm{HCl} 0,5 \%$. L'azote réduit a été déterminé selon la méthode de Kjeldahl. La conductivité électrique des extraits aqueux de sol (1 $\mathrm{g}$ de sol pour $10 \mathrm{~g}$ d'eau) a été mesurée avec un conductimètre Metrohm.

Tableau I. Caractéristiques ioniques du sol dans la zone centrale de la parcelle expérimentale.

\begin{tabular}{|c|c|c|}
\hline & $0-20$ & $20-40$ \\
\hline & $\ldots$ & $\ldots$ \\
\hline CE mS.cm-1 & $\begin{array}{l}0,13 \pm 0,01 \\
775+0,19\end{array}$ & $\begin{array}{l}0,13 \pm 0,02 \\
7,57+0,76\end{array}$ \\
\hline
\end{tabular}

\section{Paramètres}

Horizon $(\mathrm{cm})$

$\begin{array}{ccc}40-60 & 60-80 & 80-100 \\ & -\quad- & \\ 1,21 \pm 0,15 & 2,33 \pm 0,20 & 2,62 \pm 0,32 \\ 7,73 \pm 0,14 & 7,59 \pm 0,07 & 7,29 \pm 0,61\end{array}$

$\mathrm{CE}$ est la conductivité électrique de l'extrait aqueux de sol ( $1 \mathrm{~g}$ de sol pour $10 \mathrm{~g}$ d'eau). La concentration des sels totaux (méq. $\mathrm{kg}^{-1}$ de sol sec) est environ égale à CE x 100 . Moyennes et intervalles de sécurité au seuil de $95 \%$ ( 8 répétitions). 


\section{RÉSULTATS}

\section{Identification de l'écosystème}

\section{Inventaire de la végétation}

Quatre zones concentriques de superficies différentes peuvent être distinguées dans la sebkha en fonction de la nature du sol et du couvert végétal. La zone périphérique, la moins étendue, est en partie cultivée (orge, et arbustes fourragers : Acacia cyanophylla Lindl et Medicago arborea $L$ ). On y trouve également une végétation spontanée hétérogène avec une prédominance des plantes annuelles dont les graminées (Lolium rigidum Gaud, Lagurus ovatus L, Aeluropus littoralis Gouan et Cynodon dactylon $(L)$ Pers), des légumineuses ( $M$ ciliaris, $M$ truncatula, $M$ polymorpha et $M$ minima, Astragalus hamosus $\mathrm{L}$, Trifolium tomentosum $\mathrm{L}$ et Scorpiurus muricatus $L$ ), et des composées (Calendula arvensis L, Anacyclus clavatus (Desf) Pers, Pteranthus dichotomus Forsk, Pallenis spinosa (L) Cass et Scolymus hispanicus L). La plupart de ces espèces sont réputées sensibles à la salinité. Cependant Aeluropus littoralis est caractéristique d'une hydromorphie moyenne et d'une salinité allant jusqu'à 60 milliSiemens par cm (Novikoff, 1961).

Le deuxième zone, de transition entre un paysage floristiquement bien pourvu et un autre plus pauvre, est caractérisée par la présence de touffes de chénopodiacées comme Atriplex halimus $L$ et Suaeda fruticosa ( $L$ ) Forsk tolérant une gamme étendue de salinité (10 à $\left.70 \mathrm{mS} . \mathrm{cm}^{-1}\right)$. On y trouve également des espèces appartenant à des groupes écologiques caractéristiques de sols hydromorphes diversement salés, Trifolium tomentosum et Limoniastrum guyonianum Dur (sols peu salés), Hordeum maritimum With et Triglochin bulbosa $L$ (sols salés) et enfin des espèces indicatrices de faible salinité (conductivité inférieure à $25 \mathrm{mS} \mathrm{cm}^{-1}$ ) telles que $M$ ciliaris et Trifolium scabrum $\mathrm{L}$.

La troisième zone est essentiellement le lieu de développement de plantes halophiles pérennes. L'association Salsola tetandra Forsk et Suaeda fruticosa est connue pour être caractéristique d'une hydromorphie faible à moyenne dans la plus grande partie du profil, avec une conductivité variant entre 10 et $70 \mathrm{mS}^{\mathrm{cm}} \mathrm{cm}^{-1}$ (Novikoff, 1961). D'autres espèces comme Arthrocnemum indicum (Willd) Moq sont caractéristiques de sols engorgés en permanence avec une conductivité allant jusqu'à $80 \mathrm{mS} . \mathrm{cm}^{-1}$. D'autres encore sont indicatrices soit d'une grande hydromorphie (Phragmites communis Trin), soit d'une salinité très élevée (Halocnemum strobilaceum). En plus de ces halophytes pérennes nous avons noté la présence de 4 espèces du genre Medicago, de Trigonella maritima $\mathrm{Del}$ et de Trifolium tomentosum. C'est à la limite de cette zone et de la précédente que la parcelle d'étude a été délimitée. Le tableau I présente les conductivités électriques et les $\mathrm{pH}$ des extraits aqueux de sols au centre de cette parcelle. II montre que la salinité du sol est modérée dans les 40 premiers $\mathrm{cm}$, qu'elle est multipliée par 10 dès l'horizon 40-60 cm, et qu'elle continue à croître avec la profondeur en dessous de cet horizon.

La quatrième zone (centrale) a la superficie la plus importante. Elle se distingue de la précédente par la prédominance de 2 espèces indicatrices d'une hydromorphie et d'une salinité élevées (Halocnemum strobilaceum et Arthrocnemum indicum). Les touffes de la première espèce sont le lieu de développement de Spergularia salina Presl et de Trifolium tomentosum.

Les variations de la composition en espèces végétales d'une zone à l'autre permettent de déduire la présence d'un gradient croissant de salinité et d'hydromorphie en allant de la périphérie vers le centre de la dépression.

\section{Productivité de l'écosystème}

La figure $1 \mathrm{~A}$ représente les matières sèches récoltées au cours de 2 années consécutives. Deux groupes de végétaux contribuent à la production de matière sèche : les halophytes pérennes (Arthrocnemum indicum, Salsola cruciata Chevall, et Suaeda fruticosa) et le groupe de plantes annuelles comprenant essentiellement des légumineuses (Medicago), des graminées (Hordeum) et des composées. La production maximale de matière sèche de 1989 (année plus pluvieuse) est environ $150 \%$ de celle de 1988 (année sèche). Tout au long de l'année, les touffes d'halophytes pérennes présentent aussi bien des rameaux verts que des rameaux desséchés. La contribution des rameaux verts n'est prépondérante qu'au printemps, atteignant 50 à $65 \%$ chez Arthrocnemum indicum, Salsola cruciata et Suaeda fucticosa. Pour le reste de l'année, les rameaux desséchés peuvent constituer jusqu'à $75 \%$ de la matière sèche. La pluviométrie améliore la production primaire de Salsola et d'Arthrocnemum et ne modifie pas celle de Suaeda (fig 1B). Son effet est encore plus net sur 


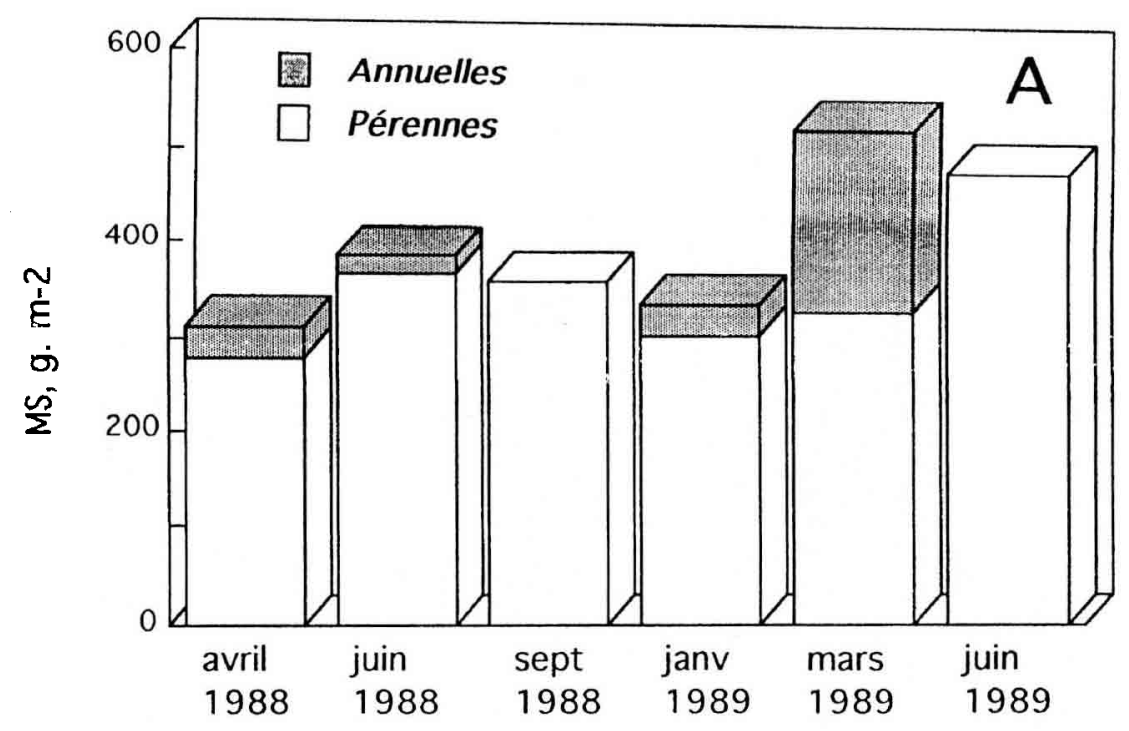

Date de prélèvement

Fig 1. Production de biomasse dans la sebkha. A. Masse de matière sèche (MS) des parties aériennes par unité de surface et par groupe d'espèces en fonction du temps. B. Production annuelle de matière sèche par unité de surface, par espèce pour les halophytes pérennes et par groupes d'espèces pour les annuelles.

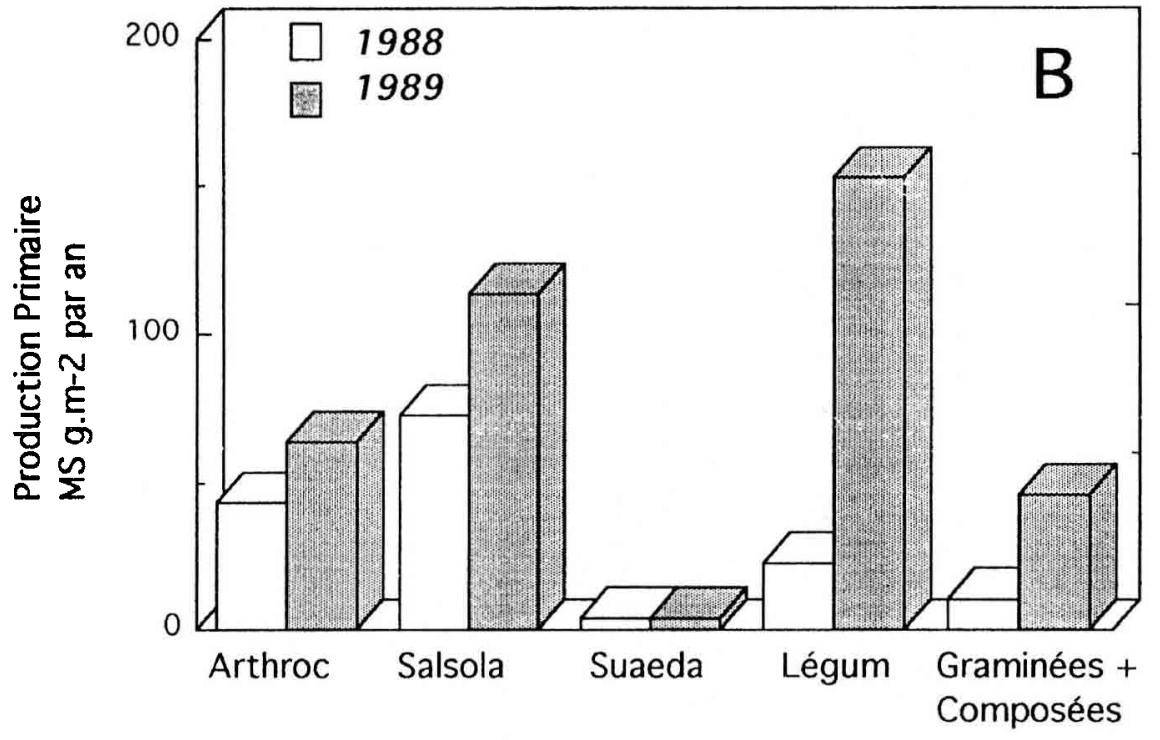

les annuelles, particulièrement les légumineuses, dont la production est multipliée par un facteur 6 au cours de l'année pluvieuse. La part de ces espèces dans la production de tout le couvert végétal (pérennes + annuelles) passe de $15 \%$ en année sèche à $40 \%$ en année humide. Parmi elles, les légumineuses occupent une place importante puisqu'elles peuvent produire jusqu'à $80 \%$ de la masse totale de ces espèces. Ces légumineuses sont représentées essentiellement par $M$ ciliaris, $M$ polymorpha, $M$ truncatula et $M$ minima.

\section{Prélèvement des éléments minéraux}

Les récoltes de printemps correspondent à un développement maximal des plantes annuelles.
En comparant, dans ces récoltes, la répartition de la MS entre les différentes espèces du couvert végétal à celle des éléments minéraux, on voit que les légumineuses sont capables d'immobiliser des quantités d'azote réduit proportionnellement plus importantes que leur MS, et inversement pour les halophytes pérennes (fig 2). Le même type de comportement est observé pour $\mathrm{K}^{+}$. Ces résultats dénotent une meilleure performance des légumineuses au niveau de l'exploitation des ressources nutritives. Chez les halophytes, $\mathrm{Na}^{+}$est surtout accumulé dans les rameaux verts où les teneurs peuvent atteindre $3 \mathrm{mmol} . \mathrm{g}^{-1} \mathrm{MS}$ ( $7 \%$ de la MS) (fig 3). Les tissus récoltés en été sont plus riches en $\mathrm{Na}^{+}$, probablement en raison de l'entraînement de ce cation dans les feuilles par le flux de transpiration. Les 


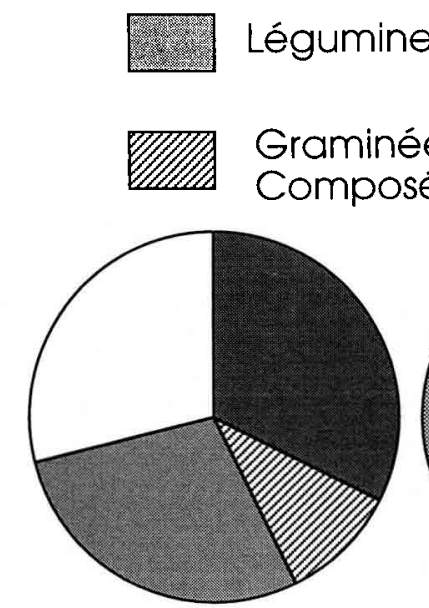

Matière sèche

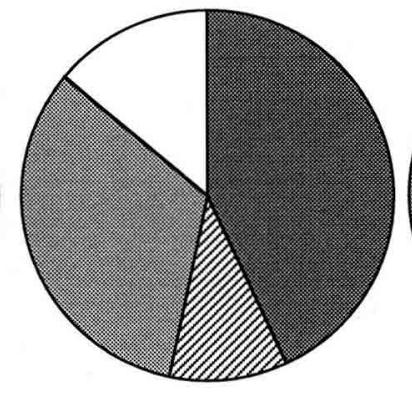

Azote
Halophytes (rameaux verts)

Halophytes (rameaux secs)

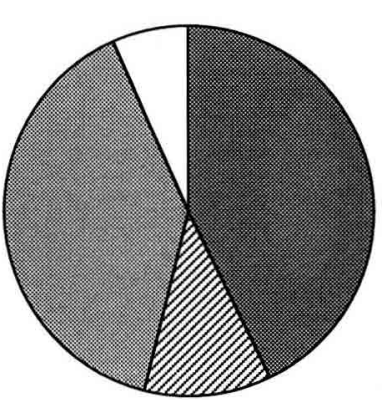

Potassium

Fig 2. Contributions relatives des groupes d'espèces à la production de biomasse et aux prélèvements d'azote et de potassium dans la sebkha. II s'agit des résultats de la récolte mars 1989. La surface de chaque secteur est proportionnelle aux quantités retrouvées dans les parties aériennes, ramenées à l'unité de surface de sol.

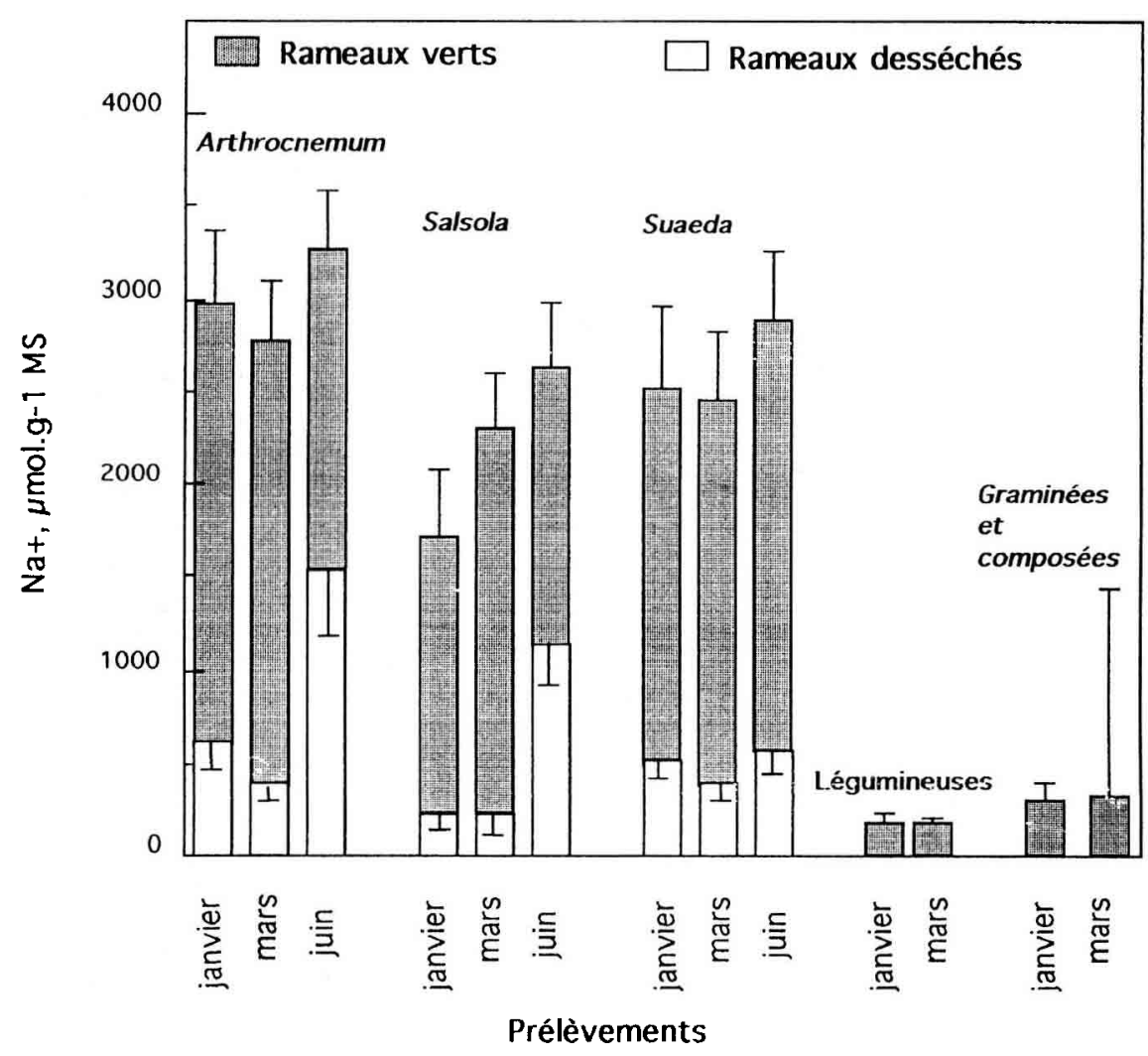

Fig 3. Teneurs en sodium des parties aériennes des différentes espèces dans la sebkha. Résultats relatifs à l'année 1989. rameaux desséchés s'appauvrissent considérablement en $\mathrm{Na}^{+}$, les niveaux d'accumulation n'étant que $20 \%$ de ceux des rameaux verts. Les annuelles accumulent peu $\mathrm{Na}^{+}$dans leurs parties aériennes $\left(0,2\right.$ à $0,3 \mathrm{mmol} . \mathrm{g}^{-1} \mathrm{MS}$ environ), ce qui suggère soit que leurs racines se développent dans des zones à faible salinité, soit qu'elles opposent une barrière efficace à l'entrée de ce cation dans la plante.

\section{Etude des effets du stress salin au laboratoire}

\section{Croissance}

Les mesures de croissance (production de matière sèche et surface foliaire) sont faites juste avant la floraison, sur des plantes cultivées en pots remplis de sol et irrigués pendant $45 \mathrm{j}$ avec les solutions T, T6 et T9. Pour une espèce don- 
née, la quantité de matière sèche déterminée à la récolte finale dépend aussi bien de la vigueur des plantes au moment de l'addition du sel que de l'activité de croissance pendant le traitement. La croissance moyenne relative (CMR), qui exprime le production de biomasse par $\mathrm{g}$ de $\mathrm{MS}$, permet d'isoler le second facteur en évaluant l'importance du ralentissement de l'activité de croissance sous l'effet du sel (fig 4). L'irrigation avec la solution T6 ne restreint pas la production de MS chez $M$ ciliaris ; elle l'inhibe de $20 \%$ chez $M$ minima, et elle a un effet intermédiaire chez les 2 autres médics. Avec la solution T9, $M$ ciliaris est la plus affectée, et c'est $M$ polymorpha qui résiste le mieux.

La réduction de la production de MS correspond à une diminution du nombre des feuilles par plante, de la surface foliaire et vraisemblablement de l'activité photosynthétique. Pour identifier le facteur le plus impliqué dans ce phénomène, nous avons calculé la vitesse d'assimilation nette par unité de surface foliaire (ANSF) et l'indice de surface foliaire (ISF). L'ANSF est la vitesse de production de biomasse par $\mathrm{cm}^{2}$ de feuilles, et l'ISF est la surface foliaire associée à $1 \mathrm{~g} \mathrm{MS}$ totale de plante. La CMR correspond généralement au produit de ces 2 paramètres (Hunt, 1990).

Le tableau II résume les valeurs moyennes des 3 paramètres CMR, ANSF et ISF, ainsi que les valeurs SF des surfaces foliaires par plante. Les CMR sont peu réduites par la salinité, surtout sur milieu T6. Chez $M$ polymorpha, $M$ ciliaris et $M$ truncatula, ce comportement est dû à une légère stimulation de l'assimilation nette par unité de surface foliaire, qui compense la baisse de surface foliaire par unité de biomasse de feuilles. II est possible que cette stimulation reflète le meilleur éclairement des feuilles résultant de la réduction de leur surface totale par plante (tableau II). Enfin, chez $M$ minima, la baisse de CMR en présence de sel est due à la forte réduction de ANSF, qui n'est qu'incomplètement compensée par une augmentation d'ISF. Cette plante présente des feuilles plus petites que celles des 3 autres espèces, et donc l'auto-ombrage dans la canopée est probablement moins important.

\section{Accumulation de $\mathrm{Na}^{+}$}

Le sodium est fortement accumulé dans tous les organes des 4 Medicago cultivés sur milieu liquide en présence de $\mathrm{NaCl}$. Les teneurs atteintes dans les parties aériennes sont de l'ordre de 1 à 2 mol. $\mathrm{g}^{-1}$ MS sur les milieux contenant $\mathrm{NaCl} 6$ et $12 \mathrm{g.l}^{-1}$ (100 et $200 \mathrm{mM}$ ) (fig 5). Ces valeurs sont 4 à 8 fois supérieures à celles que l'on observe chez les mêmes plantes récoltées dans leurs milieux naturels (fig 3 ).

\section{Production de graines et viabilité}

Chez les 4 Medicago, le sel diminue la production d'organes reproducteurs. En effet, l'indice de récolte, défini par la masse des gousses à maturité rapportée à la masse des parties aériennes à la floraison, est fortement affecté par la salinité
Fig 4. Effets de $\mathrm{NaCl}$ sur la croissance. Les plantes sont cultivées en pots, irrigués avec les solutions $\mathrm{T}, \mathrm{T} 6$ et T9 (contenant $\mathrm{NaCl} 10$, 110 et $160 \mathrm{mM}$ ). Moyennes et intervalles de sécurité au seuil $95 \%$ ( 8 répétitions). CMR désigne la croissance moyenne relative ( $\mathrm{g}$ MS produits par jour par g de MS).

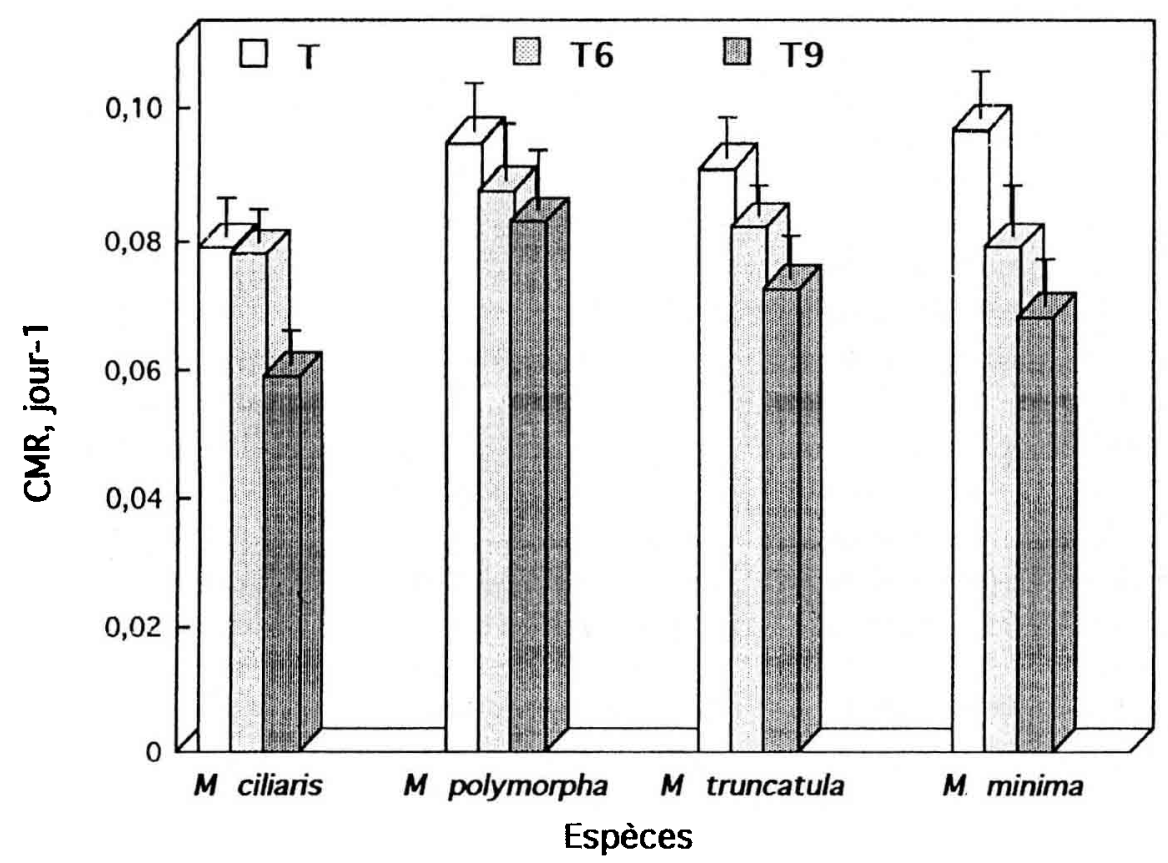


Tableau II. Décomposition de la croissance moyenne relative (CMR) des espèces de Medicago en vitesse d'assimilation nette par unité de surface foliaire (ANSF) et en indice de surface foliaire (ISF).

Espèces et paramètres

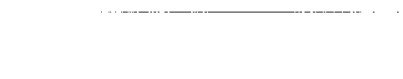

Traitement

Témoin
$0,08 \pm 0,004$
$0,256 \pm 0,054$
$262 \pm 78$
0,313

$$
\begin{gathered}
0,099 \pm 0,006 \\
0,241 \pm 0,051 \\
303 \pm 76 \\
0,411
\end{gathered}
$$
$0,092 \pm 0,004$
$0,291 \pm 0,063$
$310 \pm 82$
0,316

SF

ISF
$0,098 \pm 0,005$
$0,358 \pm 0,1$
$101 \pm 38$

SF

ISF

\section{T6}
$0,078 \pm 0,006$
$0,418 \pm 0,126$
$155 \pm 82$
0,152
$0,093 \pm 0,007$
$0,310 \pm 0,071$
$144 \pm 40$
0,300
$0,083 \pm 0,004$
$0,351 \pm 0,07$
$117 \pm 19$
0,236
$0,079 \pm 0,008$
$0,269 \pm 0,084$
$48 \pm 20$
0,297

\section{T9}

$0,060 \pm 0,004$
$0,217 \pm 0,039$
$71 \pm 13$
0,131

$0,087 \pm 0,007$

$0,272 \pm 0,073$

$118 \pm 31$

0,320
$0,071 \pm 0,007$
$0,303 \pm 0,082$
$71 \pm 19$
0,234

$0,071 \pm 0,009$

$0,229 \pm 0,082$

$43 \pm 22$

0,310

SF : surface foliaire absolue. Moyennes de 10 répétitions et intervalles de sécurité au seuil de $95 \%$.

(fig 6). Il est diminué de moitié en présence de $\mathrm{NaCl} 6 \mathrm{~g} . \mathrm{l}^{-1}$, et de 5 à 10 fois en présence de $\mathrm{NaCl} 9 \mathrm{~g} . \mathrm{l}^{-1}$. Le sel ne modifie pas la masse individuelle des graines (fig 6 , encart), mais il diminue fortement leur nombre par plante. Cette diminution résulte uniquement de la baisse du nombre de gousses, le nombre moyen de graines par gousse n'étant jamais diminué (non montré). La littérature est relativement pauvre sur ce point car la majorité des études des effets de la salinité porte sur des stades jeunes. En général, le sel semble affecter toutes les composantes du rendement à l'exception du poids de 1000 graines (Ayoub, 1977 ; lyengar et al, 1977 ; Pal et al, 1984).

Nous avons observé que les graines récoltées sur le terrain conservent une forte capacité germinative pendant au moins 6 ans. La viabilité des graines récoltées au laboratoire (expérience décrite ci-dessus) est estimée par leur capacité germinative maximale à $25^{\circ} \mathrm{C}$ sur eau distillée. Le produit de ce paramètre par le nombre de graines par plante permet d'estimer la potentialité de reproduction de chaque espèce (fig 7 ). $M$ minima ne semble pas pouvoir se reproduire en l'absence d'épisode de dessalement de son milieu, car elle ne produit pas de graines en milieu salé $\left(\mathrm{NaCl} 6 \mathrm{~g}^{-1}{ }^{-1}\right.$ ). En revanche, elle semble avoir un avantage important en absence de sel puisque notre analyse lui prévoit une descendance (nombre théorique de germinations) 2 à 6 fois plus nombreuse que celle des autres espèces. En présence de sel, l'avantage reproductif revient à $M$ polymorpha. Le dénombrement des Medicago permet d'estimer la capacité de multiplication réelle de ces plantes dans leur environnement naturel, qui est de l'ordre de 5 à 10 descendants par individu pour chacune des 4 espèces (non montré). Cette valeur est surestimée car elle ne tient pas compte de la germination des graines des générations précédentes qui auraient séjourné 1 an ou plus dans le sol avant de germer en profitant de l'année pluvieuse. Elle est très largement inférieure à la valeur théorique maximale en absence de sel, qui est de plusieurs centaines par plante d'après le décompte des graines et la mesure de leur pouvoir germinatif (fig 7). En revanche, elle est proche ( $M$ ciliaris) 
Fig 5. Accumulation de $\mathrm{Na}^{+}$dans les différents organes des Medicago. Les plantes sont cultivées pendant 3 sem en milieu hydroponique sans $\mathrm{NaCl}(T)$, puis une partie d'entre elles reçoivent $\mathrm{NaCl} 100 \mathrm{mM}$ (T6) ou $200 \mathrm{mM}$ (T12) pendant 3 autres semaines. Moyennes et intervalles de sécurité au seuil $95 \%$ ( 8 répétitions).
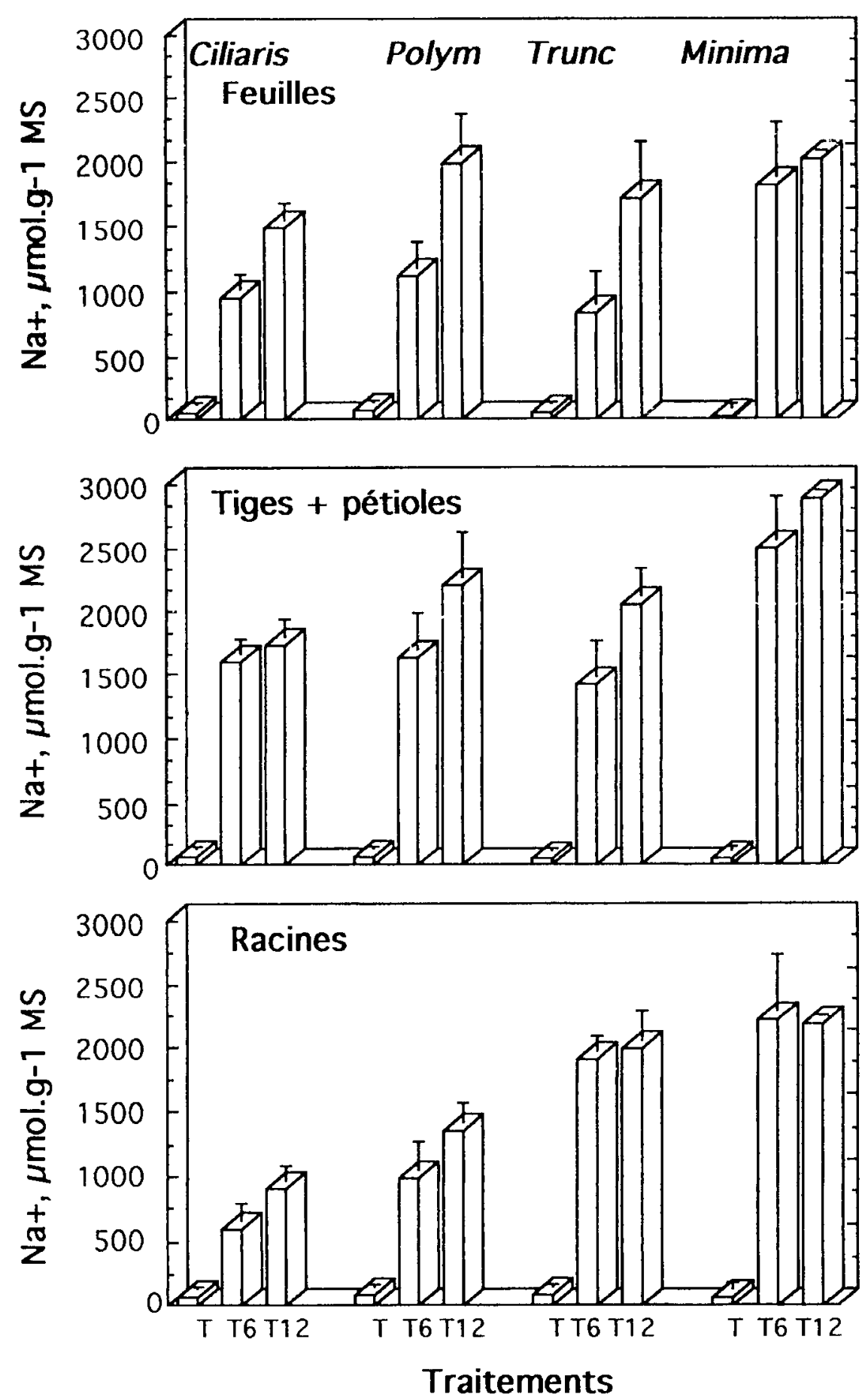

ou même supérieure ( $M$ minima) aux prévisions sur milieu salé. Nous pouvons déduire que la dynamique de reproduction du tapis végétal des légumineuses de l'écosystème dépend du maintien de la salinité à un niveau relativement faible.

\section{DISCUSSION ET CONCLUSION}

Les relevés sur le terrain montrent qu'un tapis relativement dense de plantes annuelles peut se développer rapidement entre les halophytes pérennes en bordure de sebkha, dès lors que la pluviométrie le permet. Ce tapis, qui est potentiellement intéressant en vue d'une exploitation de l'écosystème comme zone de pâturage, est composé essentiellement de 4 Medicago. Ces observations indiquent que le (micro)écosystème occupé par le tapis des légumineuses est favorable pour le développement des glycophytes, bien que la présence d'halophytes et les analyses de sol révèlent la présence de sel dans le 
sol. Nos études de laboratoire montrent que $\mathrm{NaCl}$ restreint la production de biomasse chez les 4 Medicago. Akita et Cabuslay (1990) ont montré que les réductions des valeurs de la CMR en conditions de salinité sont attribuables aux diminutions de l'ANSF et de l'ISF, sans quantifier les contributions de chacun des 2 paramètres. D'autres études avaient attribué la réduction de la croissance par le sel à une diminution de l'expansion des feuilles plutôt qu'à une baisse de leur activité de photosynthèse (ANSF), aussi bien chez un glycophyte (Curtis et Laüchli, 1986), que
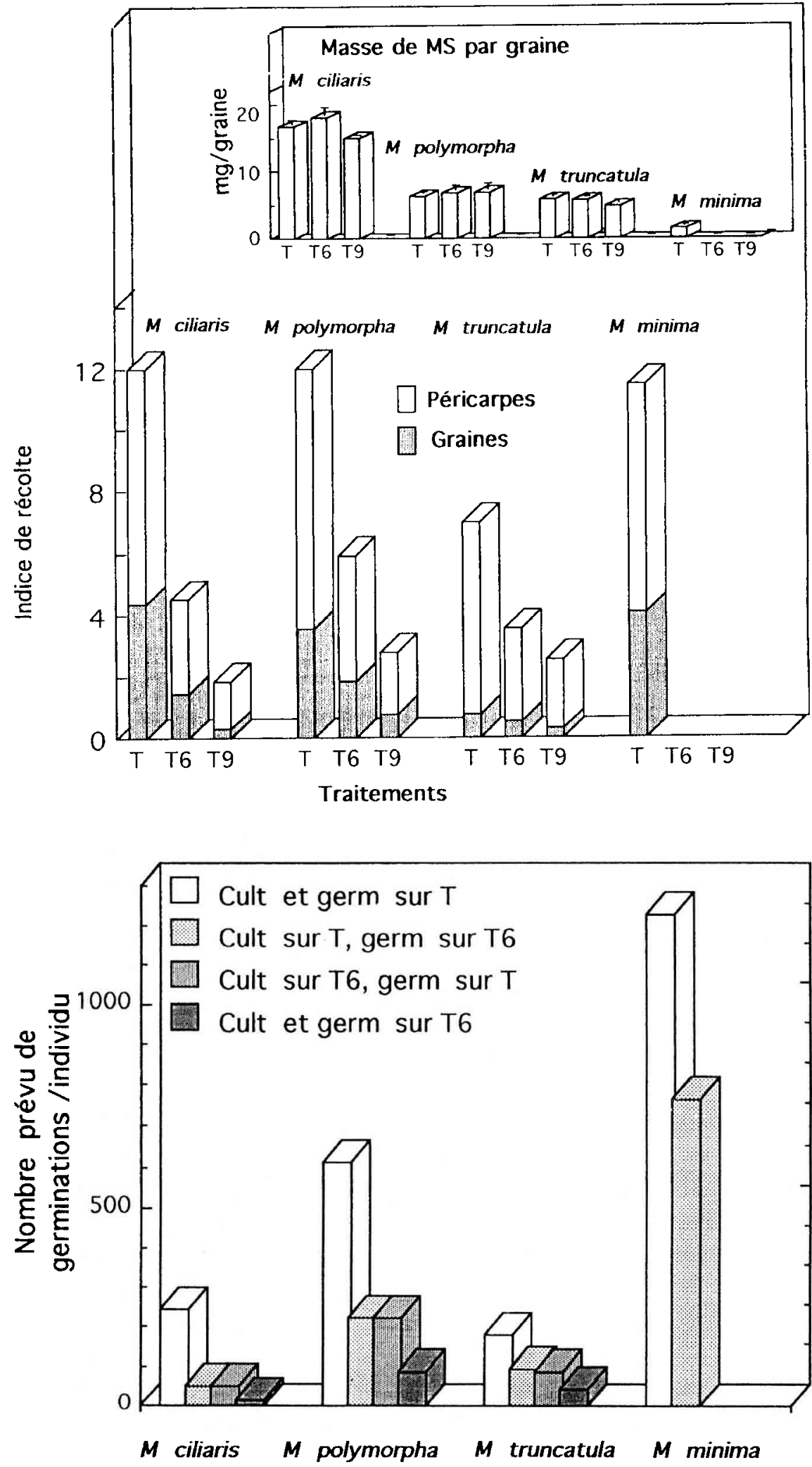

Fig 6. Production de graines. L'indice de récolte est la masse de MS de gousses ou de graines (grisé) à maturité par $\mathrm{g}$ MS de parties aériennes à la floraison. Encart: masse de MS individuelle des graines, moyennes et intervalles de sécurité au seuil $95 \%$ (10 répétitions). Les plantes sont cultivées en pots irrigués avec les solutions T, T6 ou T9.
Fig 7. Effet de $\mathrm{NaCl}$ sur la reproduction des Medicago. La potentialité de reproduction est évaluée en multipliant le nombre moyen de graines produites par plante par la capacité germinative de ces graines $(\%$ de graines capables de germer). Quatre conditions sont étudiées, différant par la présence de $\mathrm{NaCl}$ $100 \mathrm{mM}$ (T6) ou son absence $(T)$ dans les milieux de culture (et/ou) de germination. 
chez un halophyte (Shennan et al, 1987). Nos conclusions tirées de l'analyse des paramètres de la croissance des Medicago sont donc en accord avec celles de ces études.

L'opposition entre les faibles teneurs en $\mathrm{Na}^{+}$ des parties aériennes des Medicago récoltés dans leur biotope (fig 3) et les fortes accumulations de ce cation quand il est abondant dans le milieu de culture hydroponique (fig 5) est l'indice que les racines de ces plantes exploitent des horizons peu salés. L'hétérogénéité de la végétation de la station, où coexistent halophytes et glycophytes, pourrait s'expliquer par la stratification des systèmes racinaires des plantes halophiles, seules à exploiter les horizons les plus profonds et les plus salés, et des systèmes racinaires des annuelles, qui prolifèrent dans les zones superficielles moins salées (tableau I). Plusieurs hypothèses peuvent être imaginées pour expliquer le maintien d'un horizon supérieur moins salé. L'horizon supérieur peut être lessivé par une circulation latérale d'eau peu chargée vers le centre de la cuvette (hypothèse en accord avec le gradient croissant de salinité de la périphérie vers le centre de sebkhas). Les halophytes, en exploitant les horizons profonds salés, peuvent limiter la remontée de la nappe salée à la surface permettant ainsi aux eaux de pluie de pénétrer l'horizon supérieur du profil. Enfin, les halophytes pourraient favoriser le maintien de l'horizon superficiel peu salé, par désalinisation (Zhao, 1991) par leurs racines superficielles. On pourrait penser que cette désalinisation de l'horizon superficiel serait contrebalancée par la restitution d'une litière d'organes morts riches en sel ; mais nous avons constaté (résultats non publiés) que les rameaux desséchés des halophytes étudiés s'appauvrissent considéralement en $\mathrm{Na}^{+}$avant leur chute (les teneurs en $\mathrm{Na}^{+}$sont environ $20 \%$ de celle des rameaux verts). En revanche, cette litière contribue à l'enrichissement de l'horizon supérieur en nutriments, notamment l'azote (résultats non publiés). La fertilité relative de cet horizon pourrait aussi être permise par les faibles exigences de ces plantes à croissance lente (fig 1), et par leur capacité de remobiliser ou restituer au sol les éléments minéraux de leurs organes desséchés (fig 2). Ces facteurs favorisent le développement rapide d'un tapis d'annuelles peut résistantes au stress salin mais très performantes dans l'acqusition des ressources minérales dès que les ressources en eau le permettent. Ces espèces investissent fortement dans le développement reproducteur quand les conditions sont favorables (fig 6), et produisent des graines capables de conserver leur capacité germinative plusieurs années. Ce comportement est typique de la stratégie écologique dite «rudérale» (Grime, 1977), qui caractérise les plantes colonisant les milieux offrant un niveau élevé de ressources, mais présentant une forte probabilité de risque accidentel, comme ici la sécheresse.

\section{RÉFÉRENCES}

Abdelly C (1992) Réactions aux contraintes nutritionnelles des principales herbacées du tapis végétal en bordure de sebkha. Thèse de spécialité, université de Tunis, 1-252

Akita S, Cabuslay GS (1990) Physiological basis of differential response to salinity in rice cultivars. Plant Soil 123, 277-294

Ayoub AT (1977) Salt tolerance of lentil (Lens esculenta). J Hort Sci 52, 163-168

Berger A, Corre JJ, Heim G (1978) Structure, productivité et régime hydrique de phytocénoses halophiles sous climat méditerranéen. La Terre et la Vie 32 , 211-278

Curtis PS, Laüchli A (1986) The role of leaf area development and photosynthetic capacity in determining growth of kenaf under moderate salt stress. Aust $J$ Plant Physiol 18, 553-565

Epstein E (1980) Responses of plants to saline environments. In: Genetic engineering of osmoregulation: Impact on Plant Productivity for Food, Chemicals, and Energy (DW Rains, RC Valentine, A Hollaender, eds), Plenum Press, New York et Londres, 7-21

Epstein E, Norlyn JD, Rush DW, Kingsbury RW, Kelley DB, Cunningham GA, Wrona AF (1980) Saline culture of crops: a genetic approach. Science 210 , 399-404

Grime JP (1977) Evidence for existence of 3 primary strategies in plants and its relevance to ecological and evolutionary theory. Amer Naturalist 111, 11691194

Hewitt EJ (1966) Sand and Water Culture Methods Used in the Study of Plant Nutrition $\left(2^{\mathrm{e}} \mathrm{ed}\right)$. Commonwealth Bureau of Horticulture Tech Comm 22 Commonwealth Agric, Bureau Farham Royal

Hunt R (1990) Basic growth analysis. Plant growth analysis for beginners. Unwin Hyman, Londres, 1112

lyengar ERR, Patolia JS, Kurian T (1977) Varietal difference in barley to salinity. $Z$ Pflanzenphysiol $B d$ $84 \mathrm{~S}, 355-361$

Le Houérou HN (1986) Salt-tolerant plants of economic value in the Mediterranean basin. Reclamation and Revegetation Research 5, 319-341

Nasr H, Sghari R, Mhiri A, Elloumi MJ (1989) Comportement d'Acacia cyanophylla Lind dans un sol salé, résultats préliminaires. Actes du Colloque sur les végétaux en milieu aride, Tunisie (Jerba), 221-242 
Novikoff G (1961) Contribution à l'étude des relations entre le sol et la végétation halophile de Tunisie. Ann Inst Nat Agron Tunisie 34, 1-339

Pal B, Singh C, Singh H (1984) Barley yield under saline water cultivation. Plant Soil 81, 221-238

Shay EG (1990) Saline agriculture. Salt-tolerant plants for developing countries. Report of a Panel of the Board on Science and Technology for International Development Office of International Affairs, National Academy Press, Washington, DC, 1-143
Shennan C, Hunt R, MacRobbie EAA (1987) Salt tolerance in Aster tripolium L. I. The effect of salinity on growth. Plant Cell Environ 10, 59-65

Smiti S (1987) Comportement physiologique de I'Halocnemum strobilaceum (Pall) M Bied. Effet de la salinité et de l'immersion. Thèse de spécialité, université de Tunis, 1-165

Zhao KF (1991) Desalinization of saline soils by Suaeda salsa. Plant Soil 135, 303-305 